\title{
THE GENERALIZED HARMONIC BALANCE METHOD FOR DETERMINING THE COMBINATION RESONANCE IN THE PARAMETRIC DYNAMIC SYSTEMS
}

\author{
W. SZEMPLIŃSKA-STUPNICKA \\ Institute of Fundamental Technological Research of the Polish Academy of Sciences, \\ Swiętokrzyska 21, 00-049 Warsaw, Poland
}

\begin{abstract}
For a multi-degree-of-freedom system under parametric excitation an attempt is made to generalize the harmonic balance method to the case of the combination resonance. The two harmonic components solution with uncommensurable frequencies has been assumed on the stability limits. It is found that besides the condition of zero value of the characteristic determinant of the algebraic system derived by the harmonic balance procedure, the additional condition of vanishing of all minors must be satisfied. The method has been applied to a two-degree-of-freedom system. The boundaries of the principal periodic and combination resonances have been calculated theoretically and then the results have been checked by an analog computer analysis. New essential features and peculiarities of the combination resonance have been found.
\end{abstract}

\section{INTRODUCTION}

Stability analysis of the trivial solution of linear differential equations with periodic coefficients is a subject of considerable interest in the recent literature, since the problem arises naturally in the study of many physical systems.

In the field of mechanics such equations appear and need particular attention in the study of dynamic stability of structures under periodic load, the stability of machines and mechanisms with periodically varying inertia and stiffness coefficients, the attitude stability of a spinning satellite in an elliptic orbit, the aeroelastic stability of helicopter blades, the stability of periodic response of vibrating non-linear systems, the stability of pipes conveying pulsating fluids, and others [1-17]. Recent noteworthy theoretical investigations in the area have been carried out by Bolotin [16], Hsu [18], Jakubovic and Stargincky [17], Valeev [19], Schmidt $[15,20]$, Massa [21], and Nishikawa and Willems [22], and the basic concepts and earlier references can be found in the book by Cesari [23].

In the investigations of practical problems two groups of methods are in common use: the direct numerical methods based on Floquet's theorem and employing the multiple numerical integration of the differential equations for a set of various initial conditions, and the approximate analytical methods.

The purpose of the present paper is to present a new analytical approach to the investigation of the region of instability called "the combination resonance". This type of instability appears in systems with more than one degree of freedom in addition to the simple parametric resonances analogous to that in the Mathieu equation. The important role of the phenomenon of combination resonance has been emphasized in many papers [1-5].

Theoretical investigations of combination resonance can be carried out by making use of one of the perturbation methods, which of course are subject to the usual restrictions and limitations inherently associated with the concept of a "small parameter". 
Simple periodic parametric resonances, however, also can be investigated by a method which does not introduce the restrictions of the "small parameter" procedures. The method relies upon assuming the solution on the stability limits to be in the form of a Fourier series and applying the harmonic balance procedure. It is often referred to as Bolotin's method [16]. It is worth noting that the results as obtained by Bolotin's and a "small parameter" method, respectively, with both solutions of the same form as functions of time, show considerable discrepancies between one another in the case of systems with more than one degree of freedom.

The harmonic balance method has not yet been extended to the case of the combination resonance. The difficulty arises from the fact that on the boundary of this type of instability the response of the system is not periodic. It is almost periodic, consisting in the first approximation of the two harmonic components with uncommensurable frequencies.

It is the purpose of the present paper to present a new analytical approach which can be regarded as a generalization of the harmonic balance method to the case of the combination resonance: that is, to the case of the two-frequency solution. The theoretical investigations have been preceded by an analog-computer analysis which gave an insight into the peculiarities of the response of the dynamic system on the stability limits of the combination resonance and into the influence of the various parameters of the system on the unstable region.

\section{THE GENERAL EQUATIONS AND THE COMPARISON OF THE RESULTS OF THE PERTURBATION AND HARMONIC BALANCE METHODS}

Consider the dynamic system governed by the equations of motion, written in matrix form,

$$
\mathbf{A} \ddot{\mathbf{q}}+\left[\mathbf{K}+\mathbf{P}_{\mathbf{t}}(t)\right] \mathbf{q}+\mathbf{C} \dot{\mathbf{q}}=\mathbf{0},
$$

where $\mathbf{q}$ is the column matrix of the dependent variables $q_{1} q_{2}, \ldots q_{n}$ (the gencralized coordinates), $\mathbf{A}, \mathbf{K}$ and $\mathbf{C}$ are the inertia, stiffness and damping matrices, which are real, square, symmetric and positive definite. $P_{t}(t)$ is a square $n \times n$ matrix, whose elements are periodic in $t$ with period $T$, representing the parametric excitation of the system.

For the purpose of simplicity, without loss of generality, one may assume the parametric excitation to be harmonic in time, with frequency $2 v$ :

$$
\mathbf{P}_{t}(t)=\mathbf{P}_{t} \cos 2 v t,
$$

where $\mathbf{P}_{t}$ is a square matrix with constant coefficients.

When one deals with approximate analytical methods it is convenient to transform the equations of motion so that both inertia and stiffness matrices become diagonal ones. To this end one introduces the normal co-ordinates $\xi_{1}, \xi_{2}, \ldots \xi_{n}$, by means of the transformation

$$
\mathbf{q}=\mathbf{B} \boldsymbol{\xi},
$$

where $B$ is the modal matrix: that is, the matrix whose columns are the vectors of the coefficients of the normal mode shapes for the system (2.1) with $P_{t}=C=0$, so that then

$$
\mathbf{A q}+\mathbf{K q}=0 \text {. }
$$

Due to the orthogonality of the normal mode shapes the equations of motion (2.1) take the form

$$
\mathbf{M} \ddot{\xi}+(\Omega+\Lambda \cos 2 v t) \xi+\mathbf{H} \dot{\xi}=0,
$$

where $\Omega=\operatorname{diag}\left[\omega_{0 j}^{2} M_{j}\right], \mathbf{M}=\operatorname{diag}\left[M_{j}\right]$, and $A=\left[\lambda_{1 j}\right]$ is the square matrix of the parametric excitation, which proves to be a symmetric matrix in many problems of the dynamic stability 
of structures. The natural frequencies of the system $\omega_{01}, \omega_{02}, \ldots \omega_{0 n}$ are assumed to be different and uncommensurable numbers. $\mathbf{H}=\left[\beta_{i}\right]$ is the damping matrix, which in the general case is square and symmetric.

Following the usual classification, one may distinguish two types of instability of the trivial solution of equations (2.1) or (2.3), as follows.

1. There is the simple (periodic) parametric resonance which occurs in the neighbourhood of the frequencies

$$
v=\omega_{\mathrm{os}} / k, \quad k=1,2,3, \ldots, \quad s=1,2,3, \ldots n .
$$

In the stability limit the steady state response is periodic in $t$ with period $T$ equal to $2 \pi / v$ or $\pi / v$.

2. There is the combination parametric resonance, which occurs in the neighbourhood of the frequencies

$$
v=\left(\omega_{0 s}+\omega_{0_{p}}\right) / 2 k, \quad k=1,2, \ldots, \quad s, p=1,2, \ldots n, \quad s \neq p .
$$

The response on the stability limits is an almost periodic function of time. In the case of the principal resonance $(k=1)$ the solution consists of two harmonic components (in the first approximation).

The former type of instability - the simple (periodic) parametric resonance-is that known from the theory of the Mathieu equation, and there exist various approximate methods to determine the boundary of the unstable zones. The most popular and very effective method is that referred to as Bolotin's method [16]. In applying it one assumes the solution on the stability limits to be of the form of a truncated Fourier series:

or

$$
\begin{aligned}
q_{t}(t) & =\sum^{R}\left(a_{i r}^{(1)} \cos k v t+a_{i r}^{(2)} \sin k v t\right), \quad i=1,2, \ldots n . \\
r & =1,3,5, \ldots \\
r & =0,2,4, \ldots
\end{aligned}
$$

After substitution of expression (2.6) into equations (2.1) the coefficients of the individual harmonic components are equated separately to zero; this procedure is called the harmonic balance method. In the case of the principal simple resonance $(k=1$ in equation (2.4)), which plays a predominant role in practical problems, it is sufficient to take only one term in the expansion (2.6):

$$
q_{l}(t)=a_{l}^{(1)} \cos v t+a_{l}^{(2)} \sin v t \equiv a_{l} \cos \left(v t+\delta_{l}\right), \quad i=1,2, \ldots n .
$$

On applying the harmonic balance method with the solution (2.7) one arrives at a set of $2 n$ simultaneous linear algebraic homogeneous equations for $a_{i}^{(1)}, a_{i}^{(2)}, i=1,2, \ldots n$. Putting to zero the characteristic determinant, one obtains a relation between the frequency of the parametric excitation $2 v$ and the elements of the matrices $K$ and $C$ in equations (2.1), to be satisfied on the boundary of the unstable region:

$$
\check{f}(2 v, \mathbf{C}, \mathbf{K})=0 .
$$

Note that it is only the form of the solution as a function of time that has been assumed in equation (2.7), without any initial assumption about the mode of vibration; thus all the coefficients $a_{i}^{(1)}, a_{i}^{(2)}, i=1,2, \ldots n$ are treated as unknowns.

If the same approach is to be applied to the modal equations (2.3), the solution corresponding to equation (2.7) has to be

$$
\xi_{J}(t)=R_{J} \cos v t+S_{J} \sin v t, \quad j=1,2, \ldots n .
$$

with all the coefficients $R_{J j}, S_{j J}, j=1,2, \ldots n$ being unknown. Then, on substitution of expression (2.8) into equations (2.3), applying the harmonic balance method and equating the 
characteristic determinant to zero, one arrives at a relation between $2 v$ and the elements of the matrices $A$ and $H$, equivalent to equation (2.7a):

$$
\Delta_{2 n} \equiv f\left(2 v, \lambda_{11}, \lambda_{12}, \ldots \lambda_{1 n}, \lambda_{2 n}, \ldots \lambda_{n n}, \beta_{11}, \ldots \beta_{n n}\right)=0 \text {. }
$$

The boundary of the unstable region can be found by direct evaluation, the results usually being presented on the plane $\lambda_{s s}-2 v$ after fixing all the other coefficients. It is worth noticing that all the coefficients appearing in equations (2.3) are involved in the relation (2.9). This means that both the "direct" and the "coupling" parametric excitation coefficients, $\lambda_{l l}$ and $\lambda_{l j}, i \neq j$, are responsible for the shape of the unstable region.

The harmonic balance method as outlined above does not cover the case of the combination resonance. To investigate all types of parametric instabilities-both the simple and the combination resonances-one can use recently developed perturbation methods. To apply the method, one has to assume that a "small parameter" $\mu$ is associated with both the parametric excitation and the damping forces and transform equations (2.3) into

where $\mu \ll 1, \mu>0$.

$$
\mathbf{M} \ddot{\xi}+(\Omega+\mu \bar{\Lambda} \cos 2 v t) \xi+\mu \overline{\mathbf{H}} \xi=0,
$$

The starting point in the construction of the approximate solution of the system $(2.10)$ is the solution for $\mu=0$ :

$$
\xi_{j}(t)=a_{J} \cos \left(\omega_{0 J} t+\phi_{j}\right), \quad j=1,2, \ldots n,
$$

where $a_{j}$ and $\phi_{j}$ are arbitrary constants depending on the initial conditions.

A survey of the various procedures based on the concept of a "small parameter" $[17,18$, 20-25] has been presented in the author's earlier paper [26]. The following three methods have been analyzed and compared: the perturbation method based on Floquet's theorem; the method based on the asymptotic method of Krylov-Bogoliubov, and the averaging (the slowly varying coefficient) method. It has been shown that in the case of the principal resonances $(k=1)$, for both the simple and the combination type, the three methods yield identical results in the first approximation. Their common feature is the assumption that in the case of the simple periodic resonance only a single, "resonant" co-ordinate $\xi_{s}$ contributes to the response on the stability limits, all the others being equal to zero:

$$
\begin{aligned}
& \xi_{s}(t)=a_{s}^{(1)} \cos v t+a_{s}^{(2)} \sin v t \equiv a_{s} \cos \left(v t+\phi_{s}\right), v \approx \omega_{0 s}, \\
& \xi_{s}(t)=0, \quad j=1,2, \ldots s-1, s+1, \ldots \ldots .
\end{aligned}
$$

It results from this that the unstable region is determined by the relation

$$
\omega_{0 s}-\frac{\beta_{s s}}{2} \sqrt{\frac{\lambda_{s s}^{2}}{4 \beta_{s s} \omega_{0 s}^{2}}-1}<v<\omega_{0 s}+\frac{\beta_{s s}}{2} \sqrt{\frac{\lambda_{s s}^{2}}{4 \beta_{s s}^{2} \omega_{0 s}^{2}}-1}
$$

On the stability limits of the combination resonance it is assumed that two normal coordinates contribute to the solution:

$$
\begin{aligned}
& \xi_{s}(t)=a_{s} \cos \left(\omega_{s} t+\phi_{s}\right), \quad \xi_{p}(t)=a_{p} \cos \left(\omega_{p} t+\phi_{p}\right), \quad v \approx\left(\omega_{0 s}+\omega_{0_{p}}\right) / 2, \\
& \xi_{j}(t)=0, \quad j \neq s, \quad j \neq p,
\end{aligned}
$$

and the instability takes place within the frequency range

$$
\begin{gathered}
\omega_{0 s}+\omega_{0 p}-\frac{\beta_{s s}+\beta_{p p}}{2} \sqrt{\frac{\lambda_{s p} \cdot \lambda_{p s}}{4 \beta_{s s} \beta_{p p} \omega_{0 s} \omega_{0 p}}-1}<2 v<\omega_{0 s} \\
+\omega_{0 p}+\frac{\beta_{s s}+\beta_{p p}}{2} \sqrt{\frac{\lambda_{s p} \cdot \lambda_{p s}}{4 \beta_{s s} \beta_{p p} \omega_{0 s} \omega_{0 p}}-1 .}
\end{gathered}
$$


Note that in equations (2.11) and (2.13) not only the form of solution as a function of time but also the mode shape of vibration has been assumed. This becomes clear if one returns to the co-ordinates $\mathbf{q}$ by the transformation (2.2). If only a single co-ordinate $\xi_{s}$ is present (see equations (2.11)), the solution for $q_{l}(t)$ is

$$
q_{t}(t)=b_{0 i s} a_{s} \cos \left(v t+\phi_{s}\right), \quad i=1,2, \ldots n .
$$

When two co-ordinates $\xi_{s}$ and $\xi_{p}$ are assumed present in the solution (2.13), one obtains

$$
q_{i}(t)=b_{0 t s} a_{s} \cos \left(\omega_{s} t+\phi_{s}\right)+b_{0 l p} a_{p} \cos \left(\omega_{p} t+\phi_{p}\right), \quad i=1,2, \ldots n,
$$

where $b_{0 l s}, b_{0 l p}, i=1,2, \ldots n$ are the coefficients of the normal mode shapes of the conservative autonomous system

$$
\mathbf{A} \ddot{\mathbf{q}}+\mathbf{K q}=\mathbf{0} \text {. }
$$

It has been pointed out that in Bolotin's approach no initial assumption is made about the mode of vibration on the stability limits. On comparison of the results obtained from the two approaches one can notice an essential, qualitative difference: while the boundary of the unstable regions as given by Bolotin's method depend upon all the coefficients of the parametric excitation, the regions obtained by the perturbation method depend only on the "direct" parametric coefficient $\lambda_{s s}$ in the case of the simple resonance, and only on the "coupling" $\lambda_{s p}, \lambda_{p s}$ coefficients in the case of the combination resonance (see equations (2.12) and (2.14)).

Detailed analysis of a two degree-of-freedom system carried out with the aid of the analog computer provided the following information.

1. The boundary of the unstable regions, of both the simple and combination type of resonances, depends on the "direct" and the "coupling" parametric coefficients $\lambda_{l l}$ and $\lambda_{l}$, the combination resonance being appreciably sensitive to the "direct" coefficients $\lambda_{\| l}$.

2. The response on the stability limits for $\lambda_{11} \neq 0$ and $\lambda_{l s} \neq 0$ can differ considerably from that assumed in the perturbation methods (see equations (2.11) and (2.13)): in the case of the combination resonance each co-ordinate $\xi_{1}$ and $\xi_{2}$ includes two harmonic components, while in the case of the simple resonance both co-ordinates contribute in the response. The analysis covered a region of $\lambda_{l l}$ and $\lambda_{l j}$ such that the assumption about the form of solution as a function of time (single or two harmonic components predominating) was satisfied.

The theoretical calculations by the harmonic balance method for the simple resonances showed good agreement with the analog computer results.

Since in most mechanical systems the matrix $A$ in equations (2.3) includes both the diagonal "direct" terms $\lambda_{I I}$ and the "coupling" terms $\lambda_{l,}$ the idea of generalizing the harmonic balance method to the case of the combination resonance is an appealing one.

\section{THE GENERALIZED HARMONIC BALANCE METHOD}

In accordance with the arguments mentioned above, the solution on the stability limits of the combination resonance should be assumed to be of the form

$$
q_{i}(t)=a_{i s}^{(1)} \cos \omega_{s} t+a_{i s}^{(2)} \sin \omega_{s} t+a_{i p}^{(1)} \cos \omega_{p} t+a_{i p}^{(2)} \sin \omega_{p} t, \quad i=1,2, \ldots n,
$$

or, in the normal co-ordinates,

$$
\xi_{j}(t)=R_{J s} \cos \omega_{s} t+S_{j s} \sin \omega_{s} t+R_{j p} \cos \omega_{p} t+S_{j p} \sin \omega_{p} t, \quad j=1,2, \ldots n,
$$

where $a_{l s}^{(1),(2)}, a_{l p}^{(1),(2)}, i=1,2, \ldots n$, or $R_{J s}, R_{j p}, S_{J s}, S_{j p}, j=1,2, \ldots n$, are unknown coefficients. The unknown frequencies $\omega_{s}, \omega_{p}$ and the parametric frequency $2 v$ satisfy the relation

$$
2 v=\omega_{s}+\omega_{p}
$$


Formally one can apply the harmonic balance procedure by substitution of the solution (3.1), or (3.2), into equations (2.1), or (2.3), respectively and equating the coefficients of the $\cos \omega_{s} t, \sin \omega_{s} t, \cos \omega_{p} t, \sin \omega_{p} t$, separatcly to zero. Thus one arrives at a set of $4 n$ algebraic homogeneous linear equations for $a_{i s}^{(1),(2)}, a_{p}^{(1),(2)} i=1,2, \ldots n$, or $R_{j s}, S_{f_{s}}, R_{j p}, S_{f_{p}}$. Equating to zero the characteristic determinant of the system gives a relation, written here in a general form,

$$
\Delta_{4 n} \equiv f_{1}\left(\omega_{s}, \omega_{p}, \lambda_{11}, \ldots \lambda_{n n}, \beta_{11}, \ldots \beta_{n n}\right)=0
$$

In contrast to the simple resonance, equation (3.4) together with relation (3.3) does not make it possible to determine the boundary of the unstable region. There are two unknown frequencies $\omega_{s}$ and $\omega_{p}$ that have to be eliminated, and so an additional relation is indispensable.

The problem of finding the additional relation between the frequencies $\omega_{s}, \omega_{p}$ and the coefficients $\lambda_{11}, \lambda_{l,}, \beta_{l l}, \beta_{t j}$, without the help of any approximate assumption as used in the "small parameter" method, became an essential point in the task of the generalization of the harmonic balance method to the combination resonance.

A detailed consideration of the problem is presented here for the system with two degrees of freedom described by the equations of motion

$$
\begin{aligned}
& \xi_{1}+\omega_{01}^{2} \xi_{1}+\left(\lambda_{11} \xi_{1}+\lambda_{12} \xi_{2}\right) \cos 2 v t+\beta_{11} \dot{\xi}_{1}+\beta_{12} \dot{\xi}_{2}=0 \\
& \xi_{2}+\omega_{02}^{2} \xi_{2}+\left(\lambda_{21} \xi_{1}+\lambda_{22} \xi_{2}\right) \cos 2 v t+\beta_{21} \dot{\xi}_{1}+\beta_{22} \dot{\xi}_{2}=0
\end{aligned}
$$

\subsection{HARMONIC BALANCE METHOD-THE SIMPLIFIED CASE}

As a first step in the task of applying the harmonic balance method to the combination resonance, one can try to obtain a deeper insight into the peculiarities of the problem by assuming the simplified solution to be of the form of a single harmonic solution for each normal co-ordinate:

$$
\begin{gathered}
\xi_{1}(t)=R_{11} \cos \omega_{1} t+S_{11} \sin \omega_{1} t \equiv a_{1} \cos \left(\omega_{1} t+\phi_{1}\right), \\
\xi_{2}(t)=R_{22} \cos \omega_{2} t+S_{22} \sin \omega_{2} t \equiv a_{2} \cos \left(\omega_{2} t+\phi_{2}\right), \\
2 v=\omega_{1}+\omega_{2} .
\end{gathered}
$$

The essence of the harmonic balance method is that the following conditions be satisfied:

$$
\lim _{T \rightarrow \infty} \frac{1}{T} \int_{0}^{T} \varepsilon_{l}(t) \cos \omega_{\jmath} t \mathrm{~d} t=0, \quad \lim _{T \rightarrow \infty} \frac{1}{T} \int_{0}^{T} \varepsilon_{t}(t) \sin \omega_{\jmath} t \mathrm{~d} t=0, \quad i, j=1,2, \ldots n,
$$

where the $\varepsilon_{l}(t)$ are the "residuals" of equations (3.5) after substitution into them of the solution (3.6a). Simply speaking, one requires that the coefficients of $\cos \omega_{j} t, \sin \omega_{j} t, j=1,2$, in both equations $\varepsilon_{1}(t)$ and $\varepsilon_{2}(t)$ be equal to zero.

We can notice immediately that the simplified form of solution (3.6) (simplified compared to the solution (3.1)) with only four unknown coefficients $R_{11}, S_{11}, R_{22}, S_{22}$ makes it impossible to satisfy all the eight conditions (3.7). In such a case we can confine our requirement to fulfilling the conditions (3.7) only for $i=j$ (see references $[17,18]$ ):

$$
\begin{aligned}
& R_{11}\left(\omega_{01}^{2}-\omega_{1}^{2}\right)+S_{11} \beta_{11} \omega_{1}+\frac{1}{2} \lambda_{12} R_{22}=0, \quad-R_{11} \omega_{1} \beta_{11}+S_{11}\left(\omega_{01}^{2}-\omega_{1}^{2}\right)-\frac{1}{2} \lambda_{12} S_{22}=0, \\
& R_{22}\left(\omega_{02}^{2}-\omega_{2}^{2}\right)+S_{22} \omega_{2} \beta_{22}+\frac{1}{2} \lambda_{21} R_{11}=0, \quad-R_{22} \omega_{2} \beta_{22}+S_{22}\left(\omega_{02}^{2}-\omega_{2}^{2}\right)-\frac{1}{2} \lambda_{21} S_{11}=0 \text {. }
\end{aligned}
$$


To assure non-zero solutions for $R_{11}, S_{11}, R_{22}, S_{22}$ one equates to zero the characteristic determinant

$$
\Delta_{4} \equiv\left|\begin{array}{cccc}
\omega_{01}^{2}-\omega_{1}^{2} & \beta_{11} \omega_{1} & \frac{1}{2} \lambda_{12} & 0 \\
-\beta_{11} \omega_{1} & \omega_{01}^{2}-\omega_{1}^{2} & 0 & -\frac{1}{2} \lambda_{12} \\
\frac{1}{2} \lambda_{21} & 0 & \omega_{02}^{2}-\omega_{2}^{2} & \beta_{22} \omega_{2} \\
0 & -\frac{1}{2} \lambda_{21} & -\beta_{22} \omega_{2} & \omega_{02}^{2}-\omega_{2}^{2}
\end{array}\right|=0
$$

On developing the determinant one obtains a relation of the type

$$
\Delta_{4} \equiv f_{1}\left(\omega_{1}, \omega_{2}, \lambda_{12}, \lambda_{21}, \beta_{11}, \beta_{22}\right)=0
$$

The additional equation we are seeking can be found in the following way: after transposing the terms with $\lambda_{12}, \lambda_{21}$ to the right-hand sides of equations (3.8), we can square them and add the first to the second, and the third to the fourth. Thus we can arrive at a set of two equations in the squares of the amplitudes of $\xi_{1}$ and $\xi_{2}$, namely $R_{11}^{2}+S_{11}^{2}$ and $R_{22}^{2}+S_{22}^{2}$ :

$$
\begin{aligned}
& \left(R_{11}^{2}+S_{11}^{2}\right)\left[\left(\omega_{01}^{2}-\omega_{1}^{2}\right)^{2}+\beta_{11}^{2} \omega_{1}^{2}\right]-\lambda_{12}^{2}\left(S_{22}^{2}+R_{22}^{2}\right)=0, \\
& -\left(R_{11}^{2}+S_{11}^{2}\right) \frac{1}{4} \lambda_{21}^{2}+\left(R_{22}^{2}+S_{22}^{2}\right)\left[\left(\omega_{02}^{2}-\omega_{2}^{2}\right)^{2}+\beta_{22}^{2} \omega_{2}^{2}\right]=0 .
\end{aligned}
$$

It is evident that we ought to put the characteristic determinant of the transformed equations (3.10) also equal to zero, since we seek for solutions (3.6) with non-zero amplitudes $a_{1}, a_{2}$ :

$$
\left|\begin{array}{cc}
\left(\omega_{01}^{2}-\omega_{1}^{2}\right)^{2}+\beta_{11}^{2} \omega_{1}^{2}, & -\lambda_{12}^{2} \\
-\lambda \lambda_{21}^{2}, & \left(\omega_{02}^{2}-\omega_{2}^{2}\right)^{2}+\beta_{22}^{2} \omega_{2}^{2}
\end{array}\right|=0 .
$$

In this way we have obtained an additional relation between $\omega_{1}, \omega_{2}$ and the coefficients $\lambda_{12}, \lambda_{21}, \beta_{11}, \beta_{22}$, which has to be satisfied in the stability limit.

Equations (3.9) and (3.11) can be transformed into a simpler form:

$$
\begin{aligned}
& \lambda_{12} \cdot \lambda_{21}=4\left[\left(\omega_{01}^{2}-\omega_{1}^{2}\right)\left(\omega_{02}^{2}-\omega_{2}^{2}\right)+\beta_{11} \beta_{22} \omega_{1} \omega_{2}\right], \\
& \beta_{11} \omega_{1} /\left(\omega_{01}^{2}-\omega_{1}^{2}\right)=\beta_{22} \omega_{2} /\left(\omega_{02}^{2}-\omega_{2}^{2}\right), \quad \omega_{1}+\omega_{2}=2 v .
\end{aligned}
$$

The set of equations (3.12) enables us to find the boundary of the unstable region of the combination type of resonance.

It is worthwhile to compare the results obtained by the harmonic balance method (3.12) to those determined by the perturbation method (2.14), since in both cases the same form of approximate solution as a function of time has been assumed (see equations (3.6) and (2.13)). To this end let us regard the coefficients $\lambda_{i j}, \beta_{i j}$ and the deviations of the frequencies $\Delta \omega_{1}, \Delta \omega_{2}$ as small values of order $\mu$, as has been done in the perturbation method:

$$
\lambda_{l j}=\mu \overline{\lambda_{l j}}, \quad \beta_{l l}=\mu \overline{\beta_{l l}}, \quad \omega_{l}=\omega_{0 l}+\mu \Delta \bar{\omega}_{l}, \quad i, j=1,2 .
$$

Then we can substitute them into equations (3.12) and reject the terms of higher order with respect to $\mu$. After some transformation, equations (3.12) are reduced to

$$
\lambda_{12} \cdot \lambda_{21}=4 \beta_{11} \beta_{22} \omega_{01} \omega_{02}\left[1+4\left(2 v-\omega_{01}-\omega_{02}\right)^{2} /\left(\beta_{11}+\beta_{22}\right)^{2}\right]
$$

or, on solving with respect to $2 v$,

$$
\begin{gathered}
\omega_{01}+\omega_{02}-\frac{\beta_{11}+\beta_{22}}{2} \sqrt{\frac{\lambda_{12} \lambda_{21}}{4 \beta_{11} \beta_{22} \omega_{01} \omega_{02}}-1}<2 v<\omega_{01} \\
+\omega_{02}+\frac{\beta_{11}+\beta_{22}}{2} \sqrt{\frac{\lambda_{12} \lambda_{21}}{4 \beta_{11} \beta_{22} \omega_{01} \omega_{02}}-1}
\end{gathered}
$$


The relation (3.14), which determines the boundaries of the combination resonance zone, is identical with that obtained by the perturbation method (2.14).

The following conclusions can be drawn up from the above considerations.

If we use the simplified solution (3.6), we can determine the unstable region of the combination type by the harmonic balance method, because the additional equation is found as the condition of non-vanishing of the amplitudes $R_{11}^{2}+S_{11}^{2}, R_{22}^{2}+S_{22}^{2}$ in the transformed equations (3.10). However, the "direct" parametric excitation coefficients $\lambda_{11}, \lambda_{22}$ and the "coupling" damping coefficients $\beta_{12}, \beta_{21}$ are cut off in the course of the approximate procedure. Yet the harmonic balance method (equations (3.12)) can give more accurate results, because it does not necessitate use of the assumption that the parametric excitation and damping are small. For the "small" values of the parameters $\lambda_{12}, \lambda_{21}$ and $\beta_{11}, \beta_{22}$ the harmonic balance method gives identical results with that of the perturbation method.

\subsection{THE HARMONIC BALANCE METHOD-TWO-HARMONIC COMPONENTS SOLUTION}

One can now proceed to the task of the generalization of the harmonic balance method to the combination resonance. The solution, including two harmonic components, on the stability limits is assumed to be of the form (see equations (3.2))

$$
\begin{aligned}
& \xi_{1}(t)=R_{11} \cos \omega_{1} t+S_{11} \sin \omega_{1} t+R_{12} \cos \omega_{2} t+S_{12} \sin \omega_{2} t \\
& \xi_{2}(t)=R_{21} \cos \omega_{1} t+S_{21} \sin \omega_{2} t+R_{22} \cos \omega_{2} t+S_{22} \sin \omega_{2} t .
\end{aligned}
$$

Now all the eight ( $4 n$ in the case of an $n$-degree-of-freedom system) conditions of the harmonic balance (3.7) can be satisfied. On applying the usual procedure one arrives at a set of eight algebraic homogeneous equations for the unknown $R_{t}, S_{t}, i, j=1,2$ :

$$
\begin{aligned}
& R_{11}\left(\omega_{01}^{2}-\omega_{1}^{2}\right)+S_{11} \beta_{11} \omega_{1}+S_{21} \beta_{12} \omega_{1}+R_{12} \frac{1}{2} \lambda_{11}+R_{22} \frac{1}{2} \lambda_{12}=0, \\
& S_{11}\left(\omega_{01}^{2}-\omega_{1}^{2}\right)-R_{11} \beta_{11} \omega_{1}-R_{21} \beta_{12} \omega_{1}-S_{12} \frac{1}{2} \lambda_{11}-S_{22} \frac{1}{2} \lambda_{12}=0, \\
& R_{12}\left(\omega_{01}^{2}-\omega_{2}^{2}\right)+S_{12} \beta_{11} \omega_{2}+S_{22} \beta_{12} \omega_{2}+R_{11} \frac{1}{2} \lambda_{11}+R_{21} \frac{1}{2} \lambda_{12}=0, \\
& S_{12}\left(\omega_{01}^{2}-\omega_{2}^{2}\right)-R_{12} \beta_{11} \omega_{1}-R_{22} \beta_{12} \omega_{2}-S_{11} \frac{1}{2} \lambda_{11}-S_{21} \frac{1}{2} \lambda_{12}=0, \\
& R_{21}\left(\omega_{02}^{2}-\omega_{1}^{2}\right)+S_{11} \beta_{21} \omega_{1}+S_{21} \beta_{22} \omega_{1}+R_{12} \frac{1}{2} \lambda_{21}+R_{22} \frac{1}{2} \lambda_{22}=0, \\
& S_{21}\left(\omega_{02}^{2}-\omega_{1}^{2}\right)-R_{11} \beta_{21} \omega_{1}-R_{21} \beta_{22} \omega_{1}-S_{12} \frac{1}{2} \lambda_{21}-S_{22} \frac{1}{2} \lambda_{22}, \\
& R_{22}\left(\omega_{02}^{2}-\omega_{2}^{2}\right)+S_{12} \beta_{21} \omega_{2}+S_{22} \beta_{22} \omega_{2}+R_{11} \frac{1}{2} \lambda_{21}+R_{21} \frac{1}{2} \lambda_{22}=0, \\
& S_{22}\left(\omega_{02}^{2}-\omega_{2}^{2}\right)-R_{12} \beta_{21} \omega_{2}-R_{22} \beta_{22} \omega_{2}-S_{11} \frac{1}{2} \lambda_{21}-S_{21} \frac{1}{2} \lambda_{22}=0 .
\end{aligned}
$$

Then one puts the characteristic determinant equal to zero:

$$
\Delta_{\mathrm{s}}=\left|\begin{array}{cccccccc}
\omega_{01}^{2}-\omega_{1}^{2} & \beta_{11} \omega_{1} & \frac{1}{2} \lambda_{11} & 0 & 0 & \beta_{12} \omega_{1} & \frac{1}{2} \lambda_{12} & 0 \\
-\beta_{11} \omega_{1} & \omega_{01}^{2}-\omega_{1}^{2} & 0 & -\frac{1}{2} \lambda_{11} & -\beta_{12} \omega_{1} & 0 & 0 & -\frac{1}{2} \lambda_{12} \\
\frac{1}{2} \lambda_{11} & 0 & \omega_{01}^{2}-\omega_{2}^{2} & \beta_{11} \omega_{2} & \frac{1}{2} \lambda_{12} & 0 & 0 & \beta_{12} \omega_{2} \\
0 & -\frac{1}{2} \lambda_{11} & -\beta_{11} \omega_{2} & \omega_{01}^{2}-\omega_{2}^{2} & 0 & -\frac{1}{2} \lambda_{12} & -\beta_{12} \omega_{2} & 0 \\
0 & \beta_{21} \omega_{1} & \frac{1}{2} \lambda_{21} & 0 & \omega_{02}^{2}-\omega_{1}^{2} & \beta_{22} \omega_{1} & 1 \lambda_{22} & 0 \\
-\beta_{21} \omega_{1} & 0 & 0 & -\frac{1}{2} \lambda_{21} & -\beta_{22} \omega_{1} & \omega_{02}^{2}-\omega_{1}^{2} & 0 & -12 \lambda_{22} \\
\frac{1}{2} \lambda_{21} & 0 & 0 & \beta_{21} \omega_{2} & \frac{1}{2} \lambda_{22} & 0 & \omega_{02}^{2}-\omega_{2}^{2} & \beta_{22} \omega_{2} \\
0 & -\frac{1}{2} \lambda_{21} & -\beta_{21} \omega_{2} & 0 & 0 & -\frac{1}{2} \lambda_{22} & -\beta_{22} \omega_{2} & \omega_{02}^{2}-\omega_{2}^{2}
\end{array}\right|=0 \text {, }
$$

and obtains an equation of the type (3.4) with all the "direct" and the "coupling" coefficients involved:

$$
\Delta_{8} \equiv f_{1}\left(\omega_{1}, \omega_{2}, \lambda_{11}, \lambda_{12}, \lambda_{21}, \lambda_{22}, \beta_{11}, \beta_{12}, \beta_{21}, \beta_{22}\right)=0
$$


It turns out that it is not possible to transform equations (3.16) in a way analogous to that used in section 3.1: that is to reduce the number of equations by introducing the amplitudes $a_{i j} i, j=1,2$, instead of the coefficients $R_{l j}, S_{i j}, i, j=1,2$.

In the search for additional equations it is reasonable to focus one's attention on the question of how many constants in the assumed solution on the stability limits can take arbitrary values.

In the first place, one can answer this question for the case of the simple resonance. For the solution (2.8)

$$
\xi_{J}(t)=R_{J J} \cos v t+S_{J J} \sin v t, \quad j=1,2, \ldots n,
$$

the equation which determines the boundary of the unstable region is obtained by putting to zero the characteristic determinant of the set of $2 n$ algebraic equations with respect to $R_{f J}, S_{J j}$. Therefore there is only one of the $2 n$ constants $R_{J J}, S_{J}, j=1,2, \ldots n$, which can take on an arbitrary value.

The conclusion that in the case of the combination resonance two constants in the approximate solution (equations (3.2) or (3.15)) can take on arbitrary values can be proved by both physical and the theoretical arguments. For the time being, it is sufficient to show here how the conclusion follows immediately from the analysis and the construction of the additional determinant (3.11) in section 3.1 in the case of the simplified solution. It is clear that by equating to zero two characteristic determinants-one for the equations with respect to $R_{j J}, S_{J \jmath}, j=1,2$, and the other for the equations with respect to $R_{j J}^{2}+S_{J j}^{2}, j=1,2-$ two of the four coefficients $R_{11}, S_{11}, R_{22}, S_{22}$ are being treated as arbitrary.

In the theory of algebraic linear homogeneous equations the condition of linear dependence of two among $N$ unknowns is assured by equating to zero all the minors of order $N-1$ of the characteristic determinant $\Delta_{N}$. It has been proved that these are just the conditions that are satisfied when, apart from the vanishing of the characteristic determinant $\Delta_{4}$ (3.9), the additional determinant (3.11) vanishes. All the 16 minors of the determinant (3.9) have been examined carefully and it has been found that on equating one of them to zero, all the other minors can be reduced to two types, say, $\Delta_{3}^{(1)}$ and $\Delta_{3}^{(2)}$. Therefore the characteristic determinant $\Delta_{4}$ can be expressed as

$$
\Delta_{4}=A_{1} \Delta_{3}^{(1)}+A_{2} \Delta_{3}^{(2)}
$$

and then the conditions

$$
\Delta_{4}=0, \quad \Delta_{3}=0, \quad \omega_{1}+\omega_{2}=2 v,
$$

where $\Delta_{3}$ is a minor of an arbitrary element of the determinant $\Delta_{4}$ (not equal identically to zero), assure vanishing of all the 16 minors. The conditions (3.18) and (3.12) are equivalent.

If we now turn back to the general case of the two-harmonic component solution (3.15) and the set of eight equations (3.16) ( $4 n$ in an $n$-degree-of-freedom system), it is clear that the additional equation we are searching for is constructed by equating to zero a minor of order 7 $(4 n-1)$ of an arbitrary element of the characteristic determinant (3.17). Thus the set of equations that make it possible to determine the boundary of the instability of the combination type can be written in the following form:

$$
\Delta_{4 n} \equiv f_{1}\left(\omega_{s}, \omega_{p}, \boldsymbol{\Lambda}, \mathbf{H}\right)=0, \quad \Delta_{4 n-1} \equiv f_{2}\left(\omega_{s}, \omega_{p}, \boldsymbol{\Lambda}, \mathbf{H}\right)=0, \quad \omega_{s}+\omega_{D}=2 v,
$$

where $\Delta_{4 n-1}$ is a minor of an arbitrary element of the characteristic determinant $\Delta_{4 n}$ (a minor which is not equal to zero identically).

\section{NUMERICAL EXAMPLES AND ANALOG COMPUTER ANALYSIS}

The detailed calculations and the analog computer analysis have been carried out for the system with two degrees of freedom described by the equations of motion (3.5). 
To determine the boundaries of the combination resonance by the generalized harmonic alance method, the set of equations (3.19) was solved numerically. In the numerical prosdure the Gauss method was employed to develop the determinants and the Fletcherowell minimizing method to solve the set of three non-linear algebraic equations for the nknown $\omega_{1}, \omega_{2}$ and one of the coefficients $\lambda_{1 j}$ at a set of values of the frequency $2 v$.

To check the theoretical results equations (3.5) were simulated on an analog computer and 1e boundaries of the unstable region were found by adjusting the frequency $2 v$, at constant alues of the other parameters, to obtain the steady state response of the system.

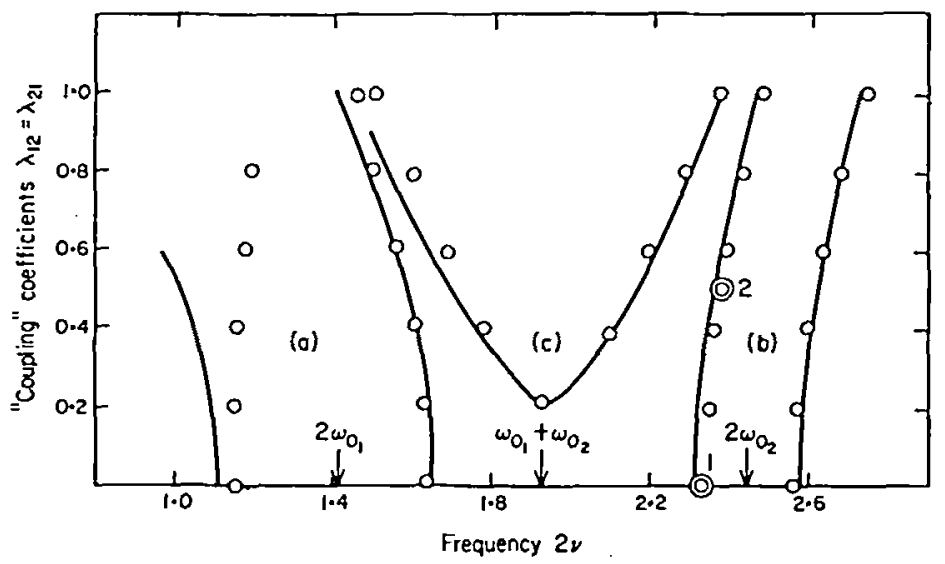

Figure 1. Instability regions of periodic (a), (b) and combination (c) resonances: $\omega_{01}^{2}=0.5, \omega_{02}^{2}=1.5$, $1=\lambda_{22}=0 \cdot 4, \beta_{12}=\beta_{21}=0 . \beta_{11}=\beta_{22}=0 \cdot 1:-$, tieoretical; 0 , analog computer results.

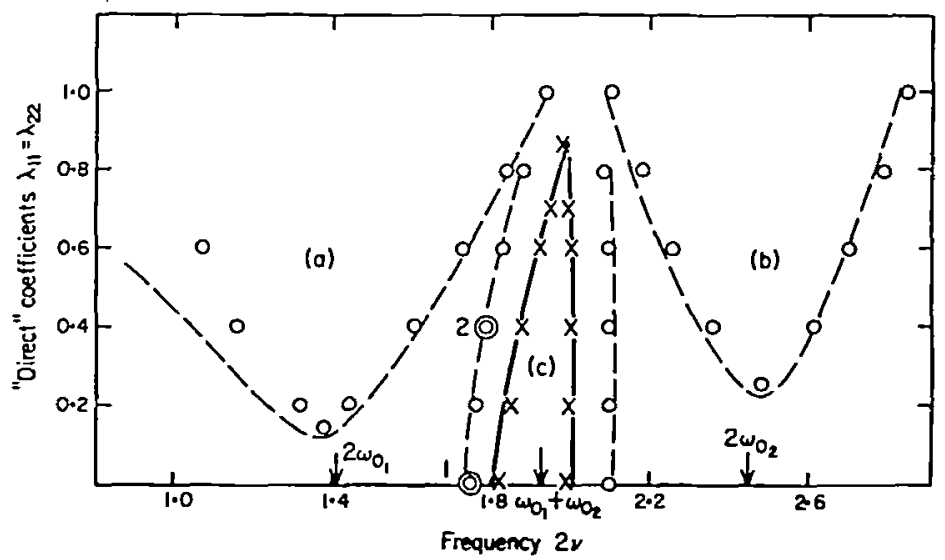

Figure 2. Instability regions of periodic (a), (b) and combination (c) resonances: $\omega_{01}^{2}=0.5, \omega_{02}^{2}=1.5$, ${ }_{2}=\lambda_{21}=0.4, \beta_{12}=\beta_{21}=0 . \beta_{11}=\beta_{22}=0 \cdot 1:---$, theoretical; $\circ \circ 0$, analog computer results. $\beta_{11}=\beta_{22}=$ 19: - theoretical; $\times \times \times$, analog computer results.

The results of the theoretical and the analog computer analyses are given in Figures 1-9. The unstable regions are presented in two configurations: on the plane $2 v-\lambda_{12}$ (Figures 1 Id 3), and on the plane $2 v-\lambda_{11}$ (Figures 2 and 4). Figures 1 and 3 show the dependence of e unstable regions on the "coupling" parametric excitation coefficients at a fixed value of e "direct" coefficients $\lambda_{11}=\lambda_{22}$. Figures 2 and 4 illustrate the effect of the "direct" sefficient $\lambda_{11}=\lambda_{22}$ at a constant value of the "coupling" coefficient $\lambda_{12}=\lambda_{21}$.

Some characteristic responses of the system on the stability limits obtained by the analog imputer analysis were recorded on the $X-Y$ recorder and are shown in Figures 5-9. The eady state response on the boundary of the combination resonance was also analysed into armonic components by means of a filter built up out of amplifiers of the computer (Figure 8 ). 


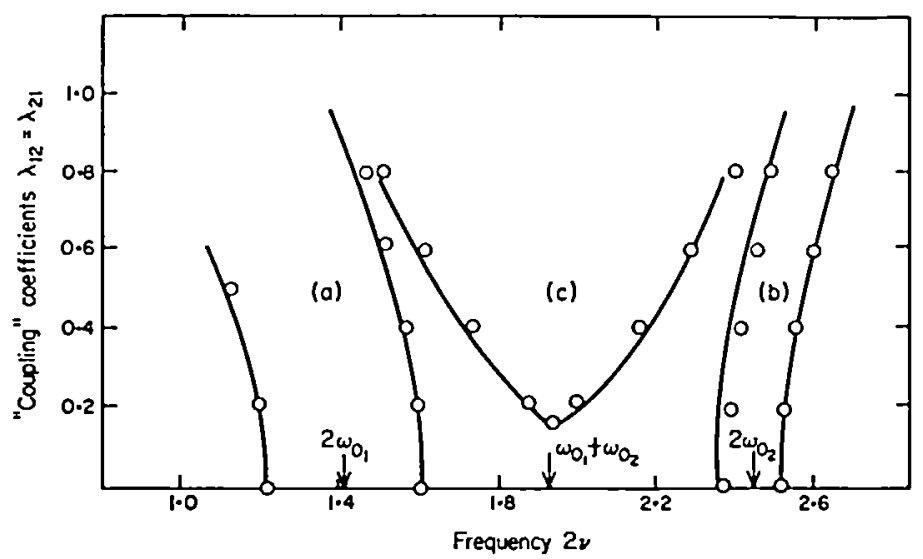

Figure 3. Instability regions of periodic (a), (b) and combination (c) resonances: $\omega_{01}^{2}=0.5, \omega_{02}^{2}=1 \cdot 5$, $\lambda_{11}=\lambda_{22}=0 \cdot 3, \beta_{12}=\beta_{21}=0 . \beta_{11}=\beta_{22}=0 \cdot 1:-$, theoretical; $\circ \circ 0$, analog computer results.

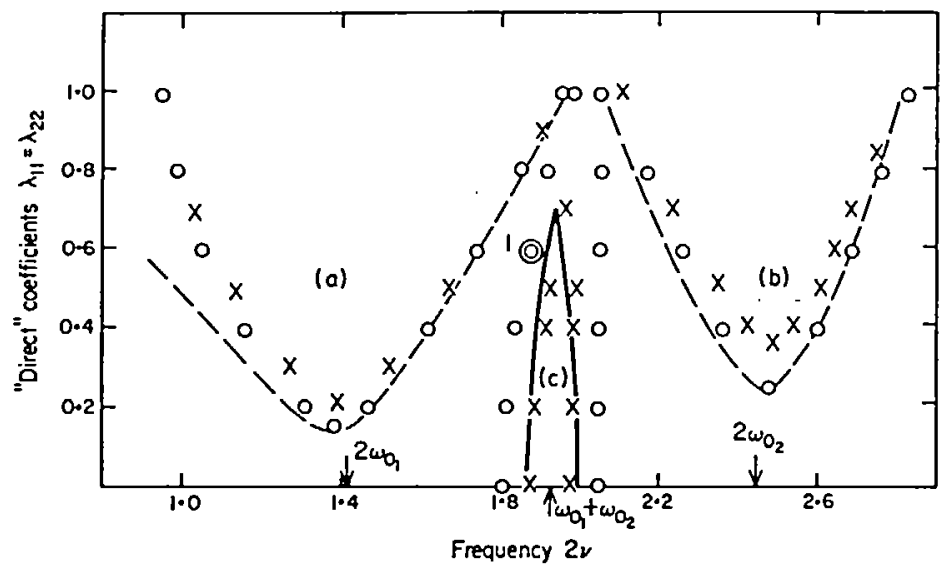

Figure 4. Instability regions of periodic (a), (b) and combination (c) resonances: $\omega_{01}^{2}=0.5, \omega_{02}^{2}=1 \cdot 5$, $\lambda_{12}=\lambda_{21}=0 \cdot 3, \beta_{12}=\beta_{21}=0 . \beta_{11}=\beta_{22}=0 \cdot 1:$ :---, theoretical; $0 \circ \circ$, analog computer results. $\beta_{11}=\beta_{22}=$ $0 \cdot 15:-$, theoretical; $\times \times \times$, analog computer results.

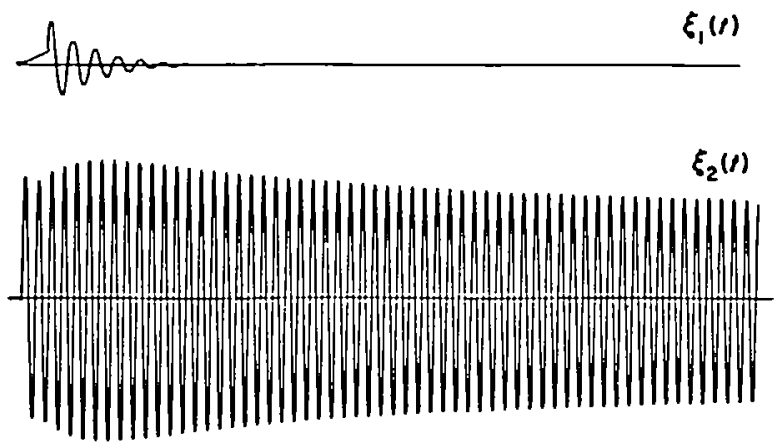

$\cos 2 v t$

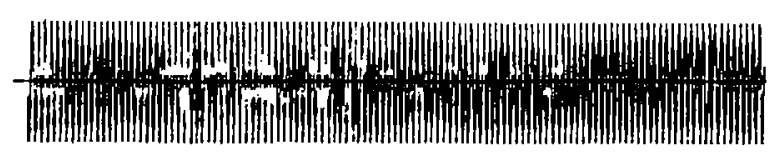

Figure 5. Wave-forms of oscillations on the boundary of the periodic resonance (point 1 in Figure 1); $\omega_{01}^{2}=0 \cdot 5, \omega_{02}^{2}=1 \cdot 5, \beta_{12}=\beta_{21}=0, \lambda_{11}=\lambda_{22}=0 \cdot 4, \lambda_{12}=\lambda_{21}=0.0$. 

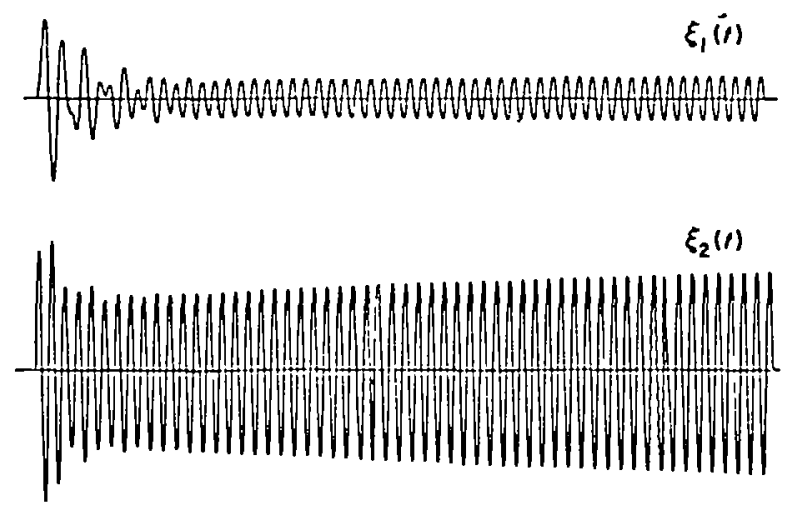

$\cos 2 v t$

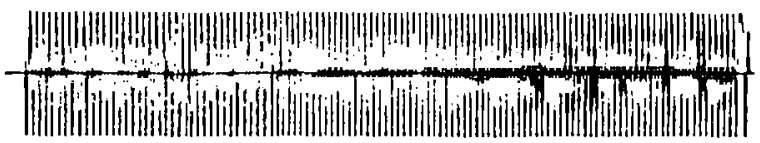

Figure 6. Wave-forms of oscillations on the boundary of the periodic resonance (point 2 in Figure 1): $\omega_{01}^{2}=0.5, \omega_{02}^{2}=1 \cdot 5, \beta_{12}=\beta_{21}=0, \lambda_{11}=\lambda_{22}=0.4, \lambda_{12}=\lambda_{21}=0.5$.

The results shown in Figures 1 and 3 and the wave-forms of oscillations in Figures 5 and 6 give an explanation of the role of the "coupling" coefficients $\lambda_{12}, \lambda_{21}$ in the case of the periodic resonances. It can be argued that the "coupling" coefficients "pull into resonance" the "non-resonant" normal co-ordinate. Only at a zero value of $\lambda_{12}$ is the "non-resonant" coordinate at rest in the steady state response. For $\lambda_{12}$ different from zero, both the "resonant" and "non-resonant" co-ordinates contribute to the periodic response. The coupling of the co-ordinates affects the frequencies of the periodic resonances: the frequency of the lower
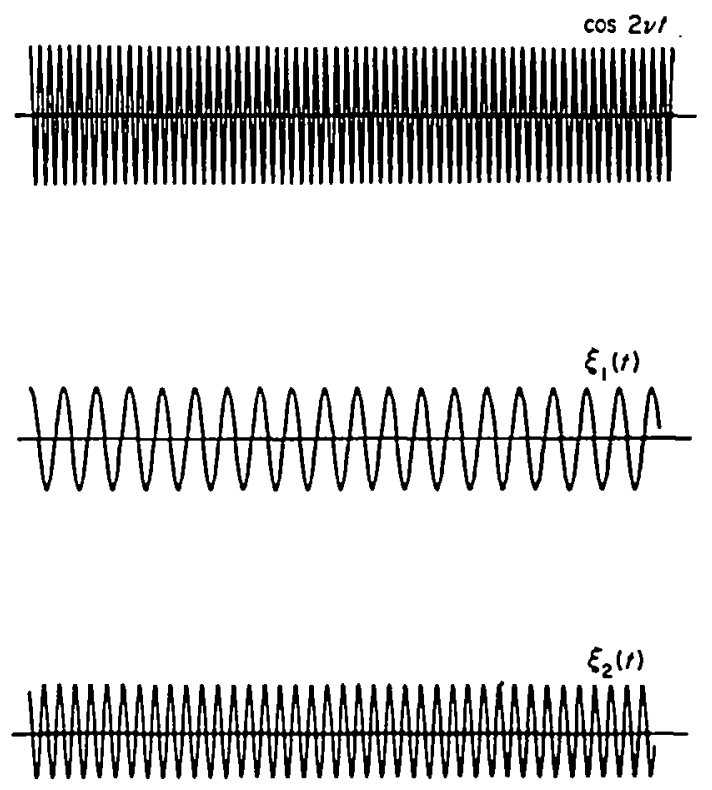

Figure 7. Wave-forms of oscillations on the boundary of the combination resonance (point 1 in Figure 2): $\omega_{01}^{2}=0.5, \omega_{02}^{2}=1.5, \beta_{12}=\beta_{21}=0, \lambda_{11}=\lambda_{22}=0.0, \lambda_{12}=\lambda_{21}=0.4$. 
$\cos 2 v t$
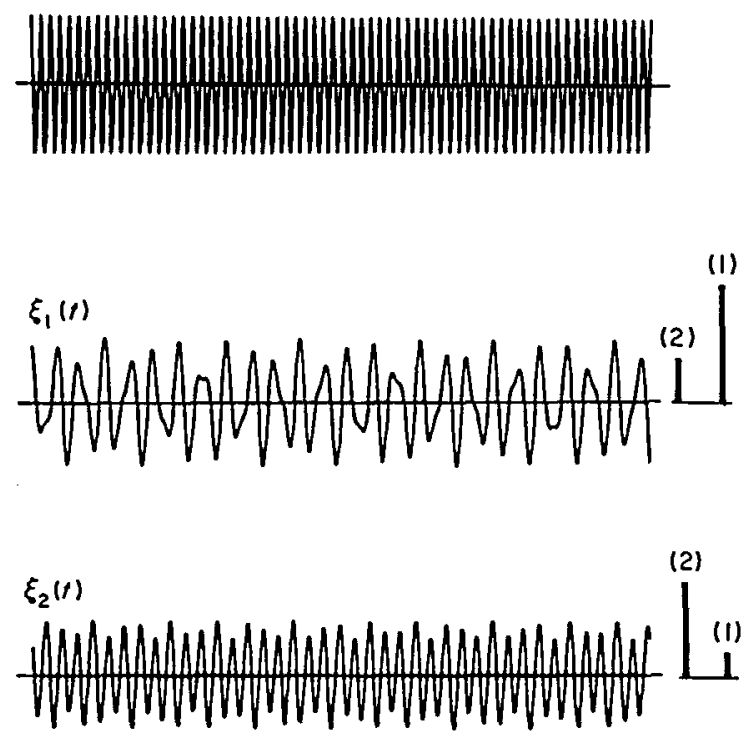

Figure 8. Wave-forms of oscillations on the boundary of the combination resonance (point 2 in Figure 2): $\omega_{01}^{2}=0.5, \omega_{02}^{2}=1 \cdot 5, \beta_{12}=\beta_{21}=0, \lambda_{11}=\lambda_{22}=0.4, \lambda_{12}=\lambda_{21}=0.4, \beta_{11}=\beta_{22}=0.1$.

periodic resonance decreases, and that of the higher frequency resonance increases with an increase of the coefficients $\lambda_{12}, \lambda_{21}$.

The unstable regions shown in Figures 2 and 4, and the response of the system in Figures 7-9 give an insight into the peculiar properties of the combination resonance. It is seen that the width of the unstable zone diminishes with an increase of the "direct" parametric excitation coefficients $\lambda_{11}=\lambda_{22}$. Above certain value of $\lambda_{11}$ the combination resonance does not exist.
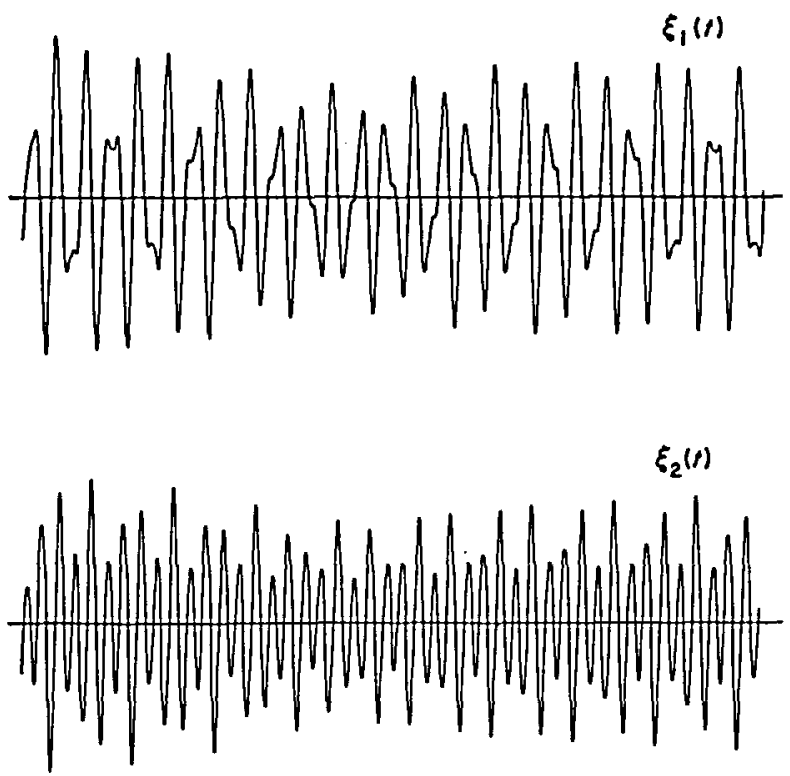

Figure 9. Wave-forms of oscillations on the boundary of the combination resonance (point 1 in Figure 4): $\omega_{01}^{2}=0.5, \omega_{02}^{2}=1 \cdot 5, \beta_{12}=\beta_{21}=0, \lambda_{11}=\lambda_{22}=0.5, \lambda_{12}=\lambda_{21}=0.3, \beta_{11}=\beta_{22}=0.1$. 
The wave-forms of oscillations on the stability limits indicate that only for a zero value of $\lambda_{11}=\lambda_{22}$ do the two co-ordinates and $\xi_{1}, \xi_{2}$ behave as being uncoupled, so that each coordinate contains a single harmonic component (Figure 6). A non-zero value of $\lambda_{11}$ results in the appearance of both harmonic components in each co-ordinate (Figures 7 and 8 ).

The results presented show good agreement of the analog computer results and those obtained by the harmonic balance method. The discrepancies in the left-hand branch of the lower periodic resonance come from the fact that the theoretical method can not take into account the influence of the higher order unstable regions.

\section{CONCLUSIONS}

The generalization, presented here, of the harmonic balance method and the analysis of the combination resonance in parametric dynamic systems lead to the following conclusions.

1. To satisfy the conditions of the harmonic balance method (3.7) the solution on the boundary of the combination resonance has to be assumed as a two-harmonic components function of time for all the co-ordinates.

2. The relations that have to be satisfied on the stability limits are derived from (a) equating to zero the characteristic determinant of the system of $4 n$ algebraic equations and (b) equating to zero a minor of an arbitrary element of the characteristic determinant. The two conditions assure the arbitrariness of two of the $4 n$ unknown coefficients in the assumed solution.

3. The harmonic balance method indicates considerable influence of the "direct" parametric excitation coefficients, disregarded in the first approximation of the perturbation method, on the regions of the combination resonance. The width of the unstable region diminishes with an increase of the "direct" coefficients, and the combination resonance disappears.

4. The theoretical and analog computer analyses show the effect of coupling of the normal co-ordinates $\xi_{1}, \xi_{2}$. In the periodic resonances the co-ordinates are coupled by means of the "coupling" parametric excitation coefficients, and in the combination resonace by means of the "direct" parametric coefficients.

\section{REFERENCES}

1. S. Ali HASAN and A. D. S. BARR 1974 Journal of Sound and Vibration 32, 25-47. Non-linear and parametric vibration of thin-walled beams of equal angle-section.

2. A. D. S. BARR and D. C. MCWhannell 1971 Journal of Sound Vibration 14, 491-509. Parametric instability of structures under support motion.

3. M. M. Ilin and K. S. KolesNikov 1969 Mekhanika Tverdovo Tela 15, 61-72. Parametric vibrations of a cantilever rod (in Russian).

4. J. Dugundu and V. Mukhopadhyay 1973 Journal of Applied Mechanics 40, 693-698. Lateral bending-torsion vibration of a thin beams under parametric excitation.

5. T. Iwatsubo, Y. Sugiyama and S. Ogino 1974 Journal of Sound and Vibration 33, 211-221. Simple and combination resonances of columns under periodic axial loads.

6. H. D. Nelson and R. A. Conover 1971 Journal of Applied Mechanics 38, 1003-1006. Dynamic instability of a beam carrying moving masses.

7. G. A. BeNEDETti 1974 Journal of Applied Mechanics 41, 1669-1672. Dynamic instability of a beam loaded by a sequence of moving mass particles.

8. T. R. KANE and P. M. BARBA 1966 Journal of Applied Mechanics 33, 402-406. Attitude stability of a spinning satellite in an elliptic orbit.

9. F. B. WALLACE and L. MeIROvitch 1967 American Institute of Acronautics and Astronautics Journal 5, 1642-1649. Attitude instability regions of a spinning symmetric satellite in an elliptic orbit.

10. P. Friedmann and J. J. SilverthoRn 1974 American Institute of Aeronautics and Astronautics Journal 12, 1559-1565. Aeroelastic stability of periodic systems with application to rotor blade flutter. 
11. M. P. Paidoussis and C. Sundararajan 1975 Journal of Applied Mechanics 42, 780-784. Parametric and combination resonances of a pipe conveying pulsating fluid.

12. Ch. SATo 1962 IRE Transactions on Circuit Theory V. CT-9, 4. Stability conditions for resonant circuit with time-variable parameters.

13. Ch. SAto 1970 Nonlinear Oscillations in Time Varying Systems. Tokyo: Keio University Publications Company.

14. Ch. SAto and Y. Nishikawa 1972 Transactions on Nonlinear Problems, The Institution of Electronic and Communication Engineers of Japan. Combination-mode parametric oscillations.

15. G. SchMIDT 1975 Parametererregte Schwingungen. VEB Deutscher Verlag der Wissenschaften, Berlin.

16. V. V. Bolotin 1964 The Dynamic Stability of Elastic Systems. Holden-Day: San Francisco.

17. V. J. JAKUBOvic and V. M. StaRgINCKY 1972 Linear Differential Equations with Periodic Coefficients (in Russian). Moscow: Nauka.

18. C.S. Hsu 1963 Journal of Applied Mechanics 30, 363-372. On the parametric excitation of dynamic systems having multiple degrees of freedom.

19. K. G. VAleEv 1963 Prikladnaia Matematika and Mekhanika 27, 1134-1142. On the danger of combination resonance (in Russian).

20. G. SCHмIDT 1973 Proceedings EQUA-DIFF 73, Bruxelles. On the dynamic stability of systems with a finite number of degrees of freedom.

21. E. MASSA 1967 Meccanica 2, 243-255. On the instability of parametrically excited two-degree-offreedom vibrating systems with viscous damping.

22. Y. NishikawA and P. V. Willews 1969 Journal of the Franklin Institute 287, 143-157. A method for stability investigation of a periodic dynamic systems with many degrees of freedom.

23. L. Cesari 1953 Asymptotic Behavior and Stability Problems in Ordinary Differential Equations. New York: Academic Press.

24. N. N. Bogoliubov and Y. A. Mitropolsky 1961 Asymptotic Methods in the Theory of Non-linear Oscillations. Hindustan Publishing Corporation, India.

25. R. A. Struble 1962 Nonlinear Differential Equations. New York: McGraw-Hill.

26. W. Szemplińska-Stupnicka 1976 Problems of Dynamic Machines. Warsaw: Ossolineum, 263-295. Approximate analytical methods in the problems of parametric instability (in Polish). 